\title{
Small Brain Vessels Disease
}

\author{
Dra Mirta D'Ambra ${ }^{1 *}$, Ilie Vasiliv ${ }^{2}$ and Mark Karindas ${ }^{3}$ \\ ${ }^{1}$ Ministry of Health (Argentina University of Buenos Aires) Executive Board member WAMS, Argentina \\ ${ }^{2}$ Vice President WAMS, Argentina \\ ${ }^{3}$ Professor President WAMS, Argentina
}

*Corresponding author: Dra Mirta D’Ambra, Ministry of Health (Argentina University of Buenos Aires) Executive Board member WAMS, Argentina

ARTICLE INFO

Received: 㹸 July 10, 2019

Published: 㗀 July 19, 2019

Citation: Dra Mirta D’Ambra, Ilie Vasiliv, Mark Karindas. Small Brain Vessels Disease. Biomed J Sci \& Tech Res 19(5)2019. BJSTR. MS.ID.003355.

\begin{abstract}
\section{Summary}

Small vessel disease is a disorder of cerebral micro vessels that causes hyperintensities of the white matter and other frequent alterations, such as small subcortical infarcts and recent lacunae, which can be seen in brain imaging studies. Despite being a frequent cause of Cerebrovascular Accident (CVA) and dementia, the underlying pathogenesis is not well understood. Several manifestations of endothelial dysfunction of brain micro vessels were identified, including blood-brain barrier dysfunction, vasodilation involvement, vessel stiffness, blood flow dysfunction and drainage of interstitial fluid and rarefaction of the white matter, ischemia, inflammation, alteration of myelin and secondary neurodegeneration. The relationships between injuries and symptoms are very variable, but they are little known. It is an important challenge to determine which vascular functions are more important in the pathogenesis, which anomalies are reversible and to which the variability of the progression of the lesions and the symptomatology is dueb
\end{abstract}

\section{Introduction}

Small vessel disease is a disorder of the small perforating arterioles, capillaries and probably cerebral venules, which causes various lesions that can be observed in the anatomopathological examination, Magnetic Resonance Imaging (MRI) or Computerized Tomography (CT) of the brain. The typical lesions of the small vessels are hyperintensities of the white matter of presumed vascular origin, lacunae, microhemorrhages, superficial siderosis, perivascular spaces and microinfarcts. Many affected people remain asymptomatic, but the increase in the number and type of injuries and the combination thereof are associated with cognitive deterioration, dementia, depression, mobility problems, increased risk of stroke (CVA) and worse evolution after a ACV. Other typical lesions of small vessel disease are small subcortical infarcts (or lagoons) and cerebral hemorrhage, which manifest with CVA. These various clinical pictures in general have been considered separately in the research and in the clinic. However, small vessel disease causes about $25 \%$ of ischemic strokes and most hemorrhagic stroke, is the most common cause of vascular dementia, often occurs with Alzheimer's disease and worsens cognitive impairment. It contributes thus to around $50 \%$ of the dementias in the whole world. Damage related to small vessel disease is not limited to visible injuries. More sensitive MRI methods show that the anatomopathological changes occur in apparently normal white matter and gray matter, which worsen as small vessel lesions increase and white matter fibers passing through visible lesions become larger. necrosis and lead to secondary degeneration of the cortex or brainstem.

\section{Some NMR Methods are Exquisitely Sensitive to Small Changes}

The vascular endothelium also affects cerebral oxygenation, transport of metabolites and interstitial fluid balance through effects on cerebral blood flow, active and passive transporters and fluid clearance, most of which depend on the interactions between endothelial cells, pericytes, astrocytes and oligodendroglia cells. The oligodendrocytes form myelin, which accelerates the conduction of axonal signals, are very interconnected and provide energy to the axons. Evidence of the disease of small vessels both sporadic. Evidence of the disease of small vessels both sporadic and monogenic suggests that dysfunctional endothelial cells can hinder 
the formation and repair of myelin in addition to causing direct damage to myelin due to dysfunction of micro vessels.

Astrocytes connect neurons with capillaries; with their podocytes the astrocytes surround the outer part of the endothelial cells at one end and their processes hold the dendrites at the other end. In the face of neuronal activity, astrocytes send signals to the endothelial cells to increase local blood flow and ensure the supply of energy. The podocytes of the astrocytes have special proteins of the water channels, called aquaporin 4. It is believed that aquaporin- 4 molecules are important in regulating the flow of fluids through the interstitial space, thus helping to maintain the interstitial space necessary for normal neuronal function. In damaged areas, the molecules of aquaporin- 4 could be relocated to the outside of the podocyte of the astrocytes as observed in the hyperintensities of the white matter.

At the tissue level, the blood brain barrier dysfunction could have several adverse effects: the spilling of fluids, proteins and other constituents of the plasma in the perivascular tissues could increase the interstitial fluid (edema) and thicken and harden the walls of the arterioles, affecting vasodilation, and the transport of oxygen and nutrients. The constituents of the blood could be harmful in many ways, among others favoring inflammation. Fibrinogen blocks the maturation of the oligodendrocyte precursor, inhibiting the maintenance and repair of myelin. It favors the formation of $\beta$-amyloid plaques and the loss of pericytes.

The perivascular fibrin deposits are increased in patients with Alzheimer's disease, providing a possible mechanistic link between small vessel disease and the pathological anatomy of Alzheimer's disease. Subtle dysfunction of the blood-brain barrier occurs with normal aging but could be accelerated by genetic predisposition [17].

\section{Conclusion}

Investigate to determine the relative contributions of the different manifestations of endothelial dysfunctions and the time elapsed until brain damage occurs in patients with small vessel disease.

a) Longitudinal studies with detailed evaluation of the risk factors and control of the lesions in order to determine the rea- sons for the great variation in the speed of progression of small vessel lesions, why some regress and why some produce secondary damage in remote areas of the brain.

b) Explanation of the role of genetic susceptibilities versus the environment and exposures to risk factors, from birth to maturity, to understand variations in the development and symptoms of small vessel disease.

c) Knowing the reasons for the variable symptomatology, the function of the cerebral reserve and the cognitive reserve, would improve the detection, the diagnosis and, in the future, would help to adapt the treatment to each patient in particular.

\section{Acknowledgement}

Many people are asymptomatic, but when the number and type of lesions increases, they are associated with cognitive deterioration, dementia, depression, mobility problems and increased risk of stroke.

\section{References}

1. Van Veluw SJ, Shih AY, Smith EE, Chen C, Schneider JA, et al. (2017) Detection, risk factors and functional consequences of cerebral microinfarcts. Lancet Neurol 16: 730-740.

2. Debette S, Schilling S, Duperron MG, Larsson SC, Markus HS (2019) Clinical significance of magnetic resonance imaging markers of vascular brain injury: a systematic review and meta-analysis. JAMA Neurol 76: 81-94.

3. Georgakis MK, Duering M, Wardlaw JM, Dichgans M (2019) WMH and long-term outcomes in ischemic stroke: a systematic review and metaanalysis. Neurology 92(12): 1298-1308.

4. Kapasi A, DeCarli C, Schneider JA (2017) Impact of multiple pathologies on the threshold for clinically overt dementia. Acta Neuropathol 134: 171-186.

5. Bos D, Wolters FJ, Darweesh SKL, Vernooij MW, de Wolf F, et al. (2018) Cerebral small vessel disease and the risk of dementia: a systematic review and meta-analysis of population-based evidence. Alzheimers Dement 14: 1482-1492.

6. Munoz Maniega S, Chappell FM, Valdes Hernandez MC, Armitage PA, Makin SD, et al. (2017) Integrity of normal-appearing white matter: influence of age, visible lesion, burden and hypertension in patients with small-vessel disease. J Cereb Blood Flow Metab 37: 644-656.

7. Baykara E, Gesierich B, Adam R (2016) A novel imaging marker for small vessel disease based on skeletonization of white matter tracts and diffusion histograms. Ann Neurol 80: 581-592.
ISSN: 2574-1241

DOI: $10.26717 /$ BJSTR.2019.19.003355

Dra Mirta D’Ambra. Biomed J Sci \& Tech Res

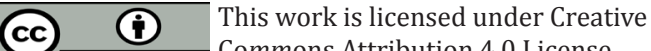

Commons Attribution 4.0 License

Submission Link: https://biomedres.us/submit-manuscript.php

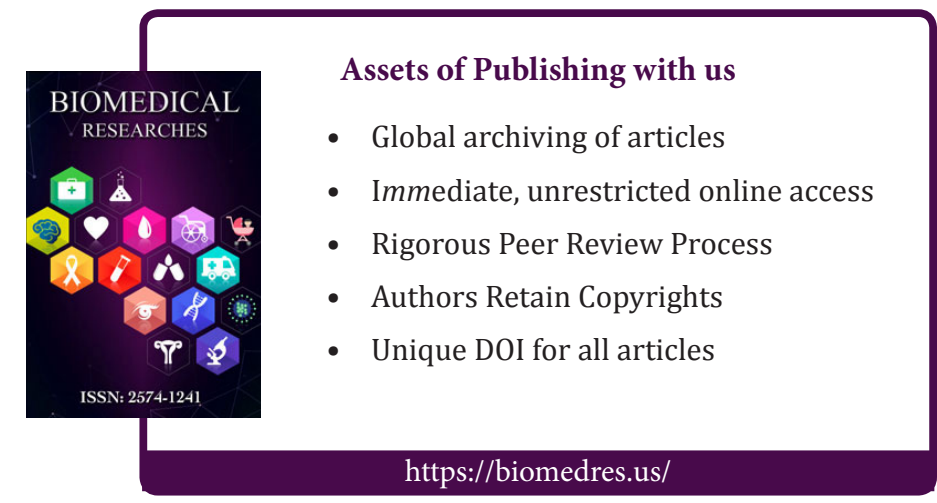

